\title{
The North American Consortium for the Study of End-Stage Liver Disease-Acute- on-Chronic Liver Failure Score Accurately Predicts Survival: An External Validation Using a National Cohort
}

\author{
Russell Rosenblatt (D) , ${ }^{1}$ Nicole Shen, ${ }^{1}$ Zaid Tafesh, ${ }^{1}$ Shirley Cohen-Mekelburg, ${ }^{2,3}$ Carl V. Crawford, ${ }^{1}$ \\ Sonal Kumar, ${ }^{1}$ Catherine Lucero, ${ }^{1}$ Robert S. Brown Jr, ${ }^{1}$ Arun Jesudian, ${ }^{1}$ and Brett E. Fortune ${ }^{1}$ \\ ${ }^{1}$ Division of Gastroenterology and Hepatology, Weill Cornell Medicine, New York, NY; ${ }^{2}$ Division of Gastroenterology and \\ Hepatology, University of Michigan Health System, Ann Arbor, MI; and ${ }^{3}$ VA Center for Clinical Management Research, \\ VA Ann Arbor Healthcare System, Ann Arbor, MI
}

\begin{abstract}
Acute-on-chronic liver failure (ACLF) carries high short-term mortality. The North American Consortium for the Study of EndStage Liver Disease (NACSELD)-ACLF score, positive if $\geq 2$ organ failures are present, is a bedside tool that predicts short-term mortality in patients with cirrhosis. However, it was created using major liver referral centers, where a minority of patients with cirrhosis are hospitalized. Therefore, this study used the Nationwide Inpatient Sample, a nationally representative database, from 2005 to 2014 to externally validate the NACSELD-ACLF score in a cohort of patients with decompensated cirrhosis who were identified by a validated algorithm. Organ failures were identified using diagnosis codes. The primary objective was to evaluate the association between the NACSELD-ACLF score and inpatient mortality, whereas secondary objectives compared outcomes depending on presence of infection or hospitalization at a transplant center. Multivariate logistic regression was used to compare outcomes, and area under the curve was calculated. There were 1,523,478 discharges that were included with 106,634 (7.0\%) having a positive NACSELD-ACLF score. Patients were a mean 58 years old, and a majority were white men. Infection was present in $33.7 \%$ of the sample. Inpatient survival decreased with each organ failure and if infection was present. Patients with the NACSELD-ACLF score had significantly lower inpatient survival on crude (94\% versus $48 \% ; P<0.001)$ and multivariate analysis (odds ratio [OR], 0.08 ; 95\% confidence interval [CI], 0.07-0.08) and area under the receiver operating characteristic curve 0.77 (95\% CI, 0.77-0.78). Liver transplant centers had clinically similar but significantly better survival at each organ failure, in patients with the NACSELD-ACLF score, and on multivariate analysis (OR, 1.17; 95\% CI, 1.13-1.22). Using a national cohort, our study validated the NACSELDACLF score as an excellent, simple bedside tool to predict short-term survival in patients with decompensated cirrhosis.
\end{abstract}

Liver Transplantation 26 187-195 2020 AASLD.

Received June 18, 2019; accepted October 26, 2019.

\section{SEE EDIIORIAL ON PAGE 179}

SEE Editorial ON PAGE 179Acute-on-chronic liver failure (ACLF) is a highly prevalent condition among

Abbreviations: ACLF, acute-on-chronic liver failure; $A U R O C$, area under the receiver operating characteristic curve; CANONIC, Chronic Liver Failure Acute-on-Chronic Liver Failure in Cirrhosis; CI, confidence interval; GI, gastrointestinal; HCUP, Healthcare Cost and Utilization Project; HE, hepatic encephalopathy; HIV, human immunodeficiency virus; ICD-9-CM, International Classification of Diseases, Ninth Revision, Clinical Modification; hospitalized patients with decompensated cirrhosis and is associated with high morbidity and mortality. Definitions of ACLF vary across different societies with European and North American consortiums generally requiring at least the presence of cirrhosis, whereas the Asian definition includes patients without cirrhosis but with chronic liver disease in addition to those with compensated cirrhosis. ${ }^{(1,2)}$ Nonetheless, ACLF is widely considered to be present when a severe acute decompensation occurs in the setting of chronic liver disease, leading to multiorgan failure. ${ }^{(1,3)}$ On the basis of this definition, studies estimate ACLF 
to be present in $30 \%$ of hospitalized patients with decompensated cirrhosis and is associated with a $34 \%$ 1-month mortality. ${ }^{(4)}$ Because of the high prevalence and short-term mortality, accurately prognosticating patients with ACLF is imperative.

Prior literature has investigated the use of scoring systems derived from prospective cohorts to prognosticate ACLF patients. First, the Chronic Liver Failure Acuteon-Chronic Liver Failure in Cirrhosis (CANONIC) group in Europe evaluated 1343 patients and established 3 prognostic stages of ACLF based on the presence of organ failures, defined by the Chronic Liver FailureSequential Organ Failure Assessment score. ${ }^{(4)}$ The North American Consortium for the Study of End-Stage Liver Disease (NACSELD), a consortium of liver referral centers from the United States and Canada, also identified the presence and number of organ failures as the key driver of mortality in hospitalized patients with cirrhosis and, thus, further refined the predictive model. ${ }^{(5)}$ More recently, the NACSELD centers reported that the presence of at least 2 organ failures (such as shock, grade 3 or 4 encephalopathy, renal failure requiring hemodialysis, or respiratory failure requiring mechanical ventilation) accurately predicted 30-day survival, which was defined as the NACSELD-ACLF score. ${ }^{(6)}$ This easily calculated bedside score theoretically enhances clinical decision making, but the generalizability of using the score is unknown.

The NACSELD cohort was derived from patients with cirrhosis from large liver transplantation (LT) referral centers, and therefore, the applicability of the

LT, liver transplantation; MELD, Model for End-Stage Liver Disease; MELD-Na, Model for End-Stage Liver Disease-sodium; NACSELD, North American Consortium for the Study of End-Stage Liver Disease; NIS, Nationwide Inpatient Sample; OR, odds ratio.

Address reprint requests to Russell Rosenblatt, M.D., Division of Gastroenterology and Hepatology, Weill Cornell Medicine, 1305 York Avenue, 4th floor, New York, NY 10021. Telephone: 646-962-5483; Fax: 646-962-0363; E-mail: RussellRosenblatt@gmail.com

Carl V. Crawford has grants from the Louis and Rachel Rudin Foundation. Arun Jesudian has stock in Salix Pharmaceuticals. Brett E. Fortune is on the speakers' bureau for W. L. Gore and Associates.

Additional supporting information may be found in the online version of this article.

Copyright (C) 2019 by the American Association for the Study of Liver Diseases.

View this article online at wileyonlinelibrary.com.

DOI 10.1002/lt.25696
NACSELD-ACLF score to the general population remains unclear. The inclusion of only LT referral centers could lead to potential bias by identifying only the sickest patients, or conversely, better LT candidates who are less frail with fewer comorbidities. When assessing the hospitalization patterns of patients with cirrhosis on a national scale, the majority are not hospitalized at a teaching or major metropolitan hospital, ${ }^{(7)}$ and may not necessarily be represented within the NACSELD cohort. Therefore, this study aimed to evaluate performance and externally validate the NACSELD-ACLF score among hospitalized patients with decompensated cirrhosis throughout the United States using a large, nationally representative database.

\section{Patients and Methods}

\section{DATA SOURCE AND PARTICIPANT SELECTION}

This study used the Nationwide Inpatient Sample (NIS; called National Inpatient Sample from 2012 to 2014) from the Healthcare Cost and Utilization Project (HCUP), Agency for Healthcare Research and Quality, from 2005 to $2014 .{ }^{(8)}$ The NIS is a large, nationally representative database of hospital discharges for all payers. The sampling methodology of the NIS changed in 2012 from a sample of $20 \%$ of hospitals participating in HCUP to a $20 \%$ sample of discharges from all participating hospitals. NIS data include demographic information, discharge disposition, diagnoses, procedures, length of stay, hospital charges, and inpatient mortality. The discharges are weighted to provide nationally representative estimates.

All discharges of patients aged 18-99 years old with both a diagnosis of cirrhosis (defined by International Classification of Diseases, Ninth Revision, Clinical Modification [ICD-9-CM] codes 571.2, 571.5, and 571.6) and the presence of a decompensating event (defined by ICD-9-CM code of bleeding esophageal varices [456.0, 456.21], spontaneous bacterial peritonitis [567.23 along with the code for paracentesis 54.91], ascites [789.5, 789.59], and hepatic encephalopathy [HE; 572.2]) were included in the study. This identification algorithm was modified from a previously validated algorithm. ${ }^{(9)}$ Our selection of a cohort of patients with decompensated cirrhosis was specifically chosen to be similar to those who were eligible for the definition of ACLF under the NACSELD cohort. ${ }^{(2)}$ Exclusion criteria were similar to that of the NACSELD studies: 
a history of prior LT, human immunodeficiency virus (HIV), actively pregnant, or extrahepatic malignancy (Supporting Materials). ${ }^{(5)}$ Additionally, patients were excluded if they underwent LT within 1 day of admission because their hospitalization would be primarily for LT and not for a complication of cirrhosis.

\section{OUTCOMES AND EXPOSURES}

Similar to the NACSELD study, ${ }^{(6)}$ organ failure was defined by the presence of shock, grade 3 or 4 encephalopathy, renal failure requiring hemodialysis, or respiratory failure requiring mechanical ventilation. Previously used algorithms were used to identify organ failures. Shock was identified by the presence of an ICD-9-CM code for septic shock (785.52), circulatory shock (785.59), or vasopressor use (00.17). ${ }^{(10)} \mathrm{HE}$ was diagnosed by the ICD-9-CM code (572.2), though unlike in the NACSELD cohort, the grade and severity could not be determined using this database. Renal failure requiring hemodialysis was diagnosed by the presence of both acute kidney injury (ICD-9-CM codes 584.5, 584.6, 584.7, 584.8, and 584.9) and hemodialysis (ICD-9-CM codes 39.95, V45.11,V56.0, and V56.1). ${ }^{(11)}$ Respiratory failure requiring mechanical ventilation was defined as the ICD-9-CM code for invasive mechanical ventilation (ICD-9-CM codes 96.04, 96.70, and 96.72) or tracheostomy placement (ICD-9-CM codes 31.1, 31.2, 31.21, and 31.29). Patients with infection were, on the basis of previous studies, ${ }^{(7,12)}$ identified as having a diagnosis of either pneumonia, urinary tract infection, Clostridium difficile infection, spontaneous bacterial peritonitis, sepsis, bacteremia, or cholangitis (Supporting Materials).

\section{OBJECTIVES}

The primary objective of the study was to evaluate the association of the NACSELD-ACLF score and inpatient survival. Secondary objectives included evaluating the impact of infection and the impact of hospital type, defined as LT or non-LT centers. LT centers were determined based on the whether an LT was performed at the hospital over the study period.

\section{STATISTICAL ANALYSIS}

STATA, version 14.0 (StataCorp, College Station, TX) was used to analyze the data. Continuous variables were expressed as means with interquartile ranges and analyzed by the adjusted Wald test. Categorical variables were expressed as percentages and analyzed by chi-square test. Multivariable logistic regression was performed to analyze outcomes. Because the NIS does not provide laboratory or vital sign information, the same model used by the NACSELD (including age, Model for End-Stage Liver Disease [MELD] score, white blood cell count, albumin, and the presence of infection) ${ }^{(6)}$ could not be used, and thus, a unique multivariable logistic regression model was constructed. The model controlled for covariates that were selected a priori and were known to impact survival in patients with cirrhosis, including age, sex, presence of infection, presence of ascites, gastrointestinal (GI) bleeding, and the Elixhauser comorbidity index. ${ }^{(13)}$ Interaction terms were included if found to be significant. Standard errors were clustered by center to limit the impact of potential outliers. The area under the receiver operating characteristic curve (AUROC) was calculated for the models. This study was deemed exempt by the Institutional Review Board at Weill Cornell Medicine.

\section{Results}

\section{PATIENT DEMOGRAPHICS}

Of the 1,612,930 discharges of patients with decompensated cirrhosis identified, 1,523,478 were included in the study with 106,634 (7.0\%) meeting the threshold for the NACSLD-ACLF score (positive when there were 2 or more organ failures; Fig. 1). The prevalence of the NACSELD-ACLF score increased from 2005 to 2014 (5.7\%-8.6\%; Supporting Table 1). Men and patients with alcohol-related liver disease were significantly more likely to have the NACSELD-ACLF score (Supporting Table 2).

The mean age was 58 years, $36.4 \%$ of patients were women, and the majority of patients were white $(65.8 \%$; Table 1). Most patients were from the lowest socioeconomic quartile, measured by median household income in the patient's ZIP code (31.9\%). Most patients were admitted in densely populated areas, and 246,124 (16.2\%) patients were hospitalized at a LT center. Alcohol-related liver disease was the most common etiology of liver disease and was significantly more common in patients with NACSELD-ACLF score. Alcoholic hepatitis, although unreliably diagnosed using ICD-9-CM codes, ${ }^{(14)}$ was present in $6.9 \%$ of the sample, and those patients had an increased prevalence of the NACSELD-ACLF score $(9.9 \%$ versus 6.7\%). Ascites (92.5\%) was the most common sign of decompensation. There were 516,089 


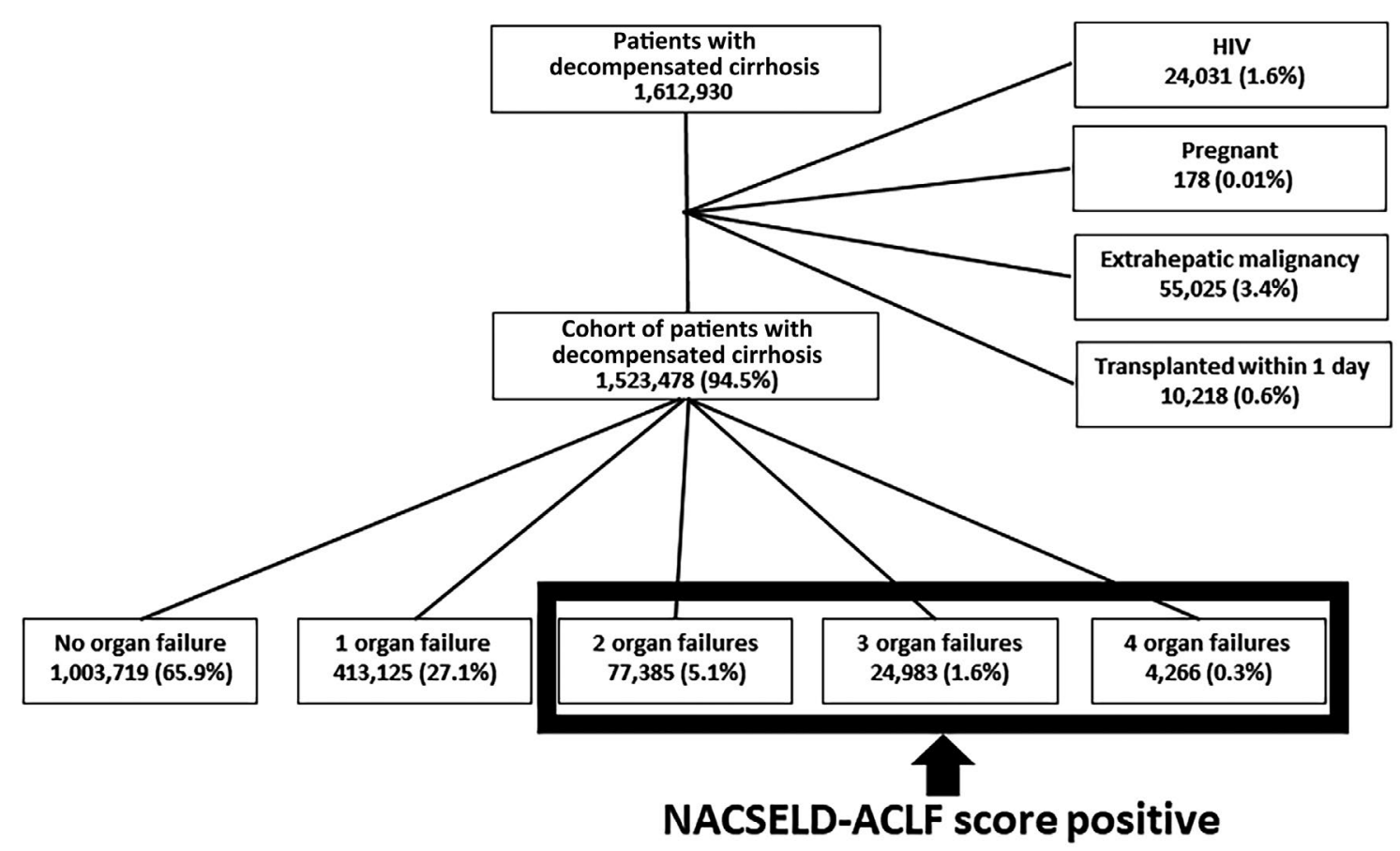

FIG. 1. Inclusion and exclusion of the study cohort.

(33.7\%) patients who were infected during their hospitalization. The most common infection was a urinary tract infection 190,792 (12.4\%) followed by pneumonia (7.0\%), spontaneous bacterial peritonitis (5.2\%), cellulitis and soft tissue infection (5.2\%), C. difficile infection $(2.3 \%)$, and cholangitis (0.7\%). Of the patients with infection, $11.9 \%$ had sepsis or septicemia. Of patients with an infection, $38.4 \%$ had at least 2 infections.

\section{CRUDE SURVIVAL}

Overall, 1,386,365 (91\%) patients survived their hospitalization during the study. Patients with the NACSELDACLF score had significantly lower survival (48\% versus 94\%; $P<0.001$; Fig. 2A). Survival in patients with the NACSELD-ACLF score significantly improved from 2005 to 2014 (38\%-53\%; Supporting Table 1). Survival was also inversely correlated with the number of organ failures, starting at $97 \%$ survival for those without organ failure and decreasing to $25 \%$ for those with 4 organ failures (Fig. 2B). At each level of organ failure, infected patients had significantly worse inpatient survival. Patients with multiple infections had higher rates of multiple organ failures (32\% versus $5 \% ; P<0.001)$ and worse overall survival (68\% versus 93\%; $P<0.001)$ than those with a single infection.
When evaluating survival rates of specific organ failures, patients with infection had higher prevalence and worse survival for each specific organ failure (Fig. $2 \mathrm{C})$. Overall, $\mathrm{HE}$ was the most common (26.6\% prevalence) organ failure but also had the highest associated survival (86.0\%). Of the individual organ faliures, shock and respiratory failure had the lowest inpatient survival (48.3\% and $47.1 \%$, respectively).

\section{REGRESSION ANALYSIS}

Univariate logistic regression demonstrated that an increasing number of organ failures was associated with decreased odds of inpatient survival (Table 2). Once at least 2 organ failures, or the NACSELD-ACLF score, was present, the combination of organ failures did not substantially change survival (Supporting Table 3). In the multivariate analysis controlling for age, sex, the interaction of GI bleeding and presence of ascites, presence of infection, and the Elixhauser comorbidity index, the NACSELD-ACLF score was significantly associated with a marked reduction in inpatient survival (adjusted odds ratio [OR], 0.08; 95\% confidence interval [CI], $0.07-0.08 ; P<0.001 ;$ Table 3). In this multivariate model, the presence of infection (adjusted OR, 0.42; 95\% CI, 0.41-0.43; $P<0.001)$ and the interaction of 
TABLE 1. Demographic Characteristics of the Sample Comparing Patients Who Meet the NACSELD-ACLF Score With Those Who Do Not Meet the Criteria

\begin{tabular}{|c|c|c|c|}
\hline Variable & Total $(n=1,523,478)$ & $\begin{array}{c}\text { NACSELD-ACLF }(n=106,634 \\
7.0 \%)\end{array}$ & $\begin{array}{c}\text { No NACSELD-ACLF } \\
(n=1,416,844,93.0 \%)\end{array}$ \\
\hline Age, years & $58 \pm 0.02$ & $56 \pm 0.08$ & $58 \pm 0.02$ \\
\hline Sex, female & $554,546(36.4)$ & $43,826(41.1)$ & $510,720(33.8)$ \\
\hline \multicolumn{4}{|l|}{ Race } \\
\hline White & $1,002,449(65.8)$ & $67,500(63.3)$ & $934,949(66.0)$ \\
\hline Black & $152,348(10.0)$ & $9,237(9.6)$ & $142,611(10.1)$ \\
\hline Hispanic & $269,656(17.7)$ & $18,727(18.5)$ & $250,729(17.7)$ \\
\hline Asian & $31,993(2.1)$ & $2,566(2.5)$ & $29,427(2.1)$ \\
\hline Other & $67,032(4.4)$ & $7,904(7.4)$ & $59,128(4.2)$ \\
\hline \multicolumn{4}{|l|}{ Median household income by ZIP code quartile } \\
\hline First & $485,989(31.9)$ & $32,050(30.1)$ & $453,939(32.0)$ \\
\hline Second & $400,675(26.3)$ & $26,888(25.2)$ & $373,787(26.4)$ \\
\hline Third & $358,017(23.5)$ & $25,501(23.9)$ & $332,516(23.1)$ \\
\hline Fourth & $278,797(18.3)$ & $22,195(20.8)$ & $256,602(18.1)$ \\
\hline \multicolumn{4}{|l|}{ Hospital location* } \\
\hline Metropolitan city center, $>1$ million & $527,123(34.6)$ & $39,948(37.5)$ & $487,175(34.4)$ \\
\hline Metropolitan county (not center), $>1$ million & $344,306(22.6)$ & $24,612(23.1)$ & $319,694(22.6)$ \\
\hline County population, 250,000-999,999 & $292,508(19.2)$ & $19,494(18.3)$ & $273,014(19.3)$ \\
\hline County population, 50,000-249,999 & $129,496(8.5)$ & $8,307(7.8)$ & $121,189(8.6)$ \\
\hline Micropolitan county & $143,207(9.4)$ & $8,841(8.3)$ & $134,366(9.5)$ \\
\hline Other & $86,838(5.7)$ & $5,432(5.1)$ & $81,406(5.7)$ \\
\hline LT hospital & $246,124(16.2)$ & $25,379(23.8)$ & $220,745(15.6)$ \\
\hline Infection & $516,089(33.7)$ & $75,710(71.0)$ & $440,379(30.9)$ \\
\hline Upper GI bleed & $278,796(18.3)$ & $37,109(34.8)$ & $241,687(17.1)$ \\
\hline Esophageal variceal bleed & $132,543(8.7)$ & $18,448(17.3)$ & $114,095(8.1)$ \\
\hline Ascites & $1,409,217(92.5)$ & $89,999(84.4)$ & $1,319,218(93.1)$ \\
\hline \multicolumn{4}{|l|}{ Type of cirrhosis } \\
\hline Hepatitis C & $457,043(30.0)$ & $20,900(19.6)$ & $436,143(30.8)$ \\
\hline Alcohol-related & $856,195(56.2)$ & $70,805(66.4)$ & $785,390(55.5)$ \\
\hline Length of stay, days & $7.0 \pm 0.01$ & $14.5 \pm 0.10$ & $6.5 \pm 0.01$ \\
\hline Total charge, US \$ & $54,920 \pm 172$ & $176,070 \pm 1525$ & $45,933 \pm 130$ \\
\hline Inpatient survival & $1,386,365(91.0)$ & $50,971(47.8)$ & $1,335,394(94.2)$ \\
\hline
\end{tabular}

NOTE: Data are given as mean \pm standard deviation or $\mathrm{n}(\%)$.

*Only variable that was not significant $(P<0.05)$. Only the two most common etiologies of cirrhosis are listed. Hepatitis $\mathrm{C}$ and Alcoholrelated are not mutually exclusive.

GI bleeding and ascites (adjusted OR, 0.78; 95\% CI, $0.73-0.84 ; P<0.001)$ were both significantly associated with reduced inpatient survival. The AUROC for the NACSELD was 0.77 (95\% CI, 0.77-0.78).

\section{LT CENTER}

Of the 246,124 patients hospitalized at a LT center, 7654 (3.1\%) underwent LT during their hospitalization. Patients who were hospitalized at an LT center were more likely to have at least 1 organ failure (66\% versus $62 \%$; $P<0.001)$ but were less likely to have the NACSELDACLF score (6\% versus 10\%; $P<0.001$ ). Survival at each number of organ failure (Fig. 3A) was significantly higher in LT centers compared with non-LT centers $(P<0.001)$. This remained true even when excluding any patient who underwent an LT. Survival from respiratory failure was significantly higher in LT centers (48\% versus 47\%; $P<0.001$ ), whereas survival from shock $(50 \%$ versus $44 \% ; P<0.001)$ and renal failure $(63 \%$ 

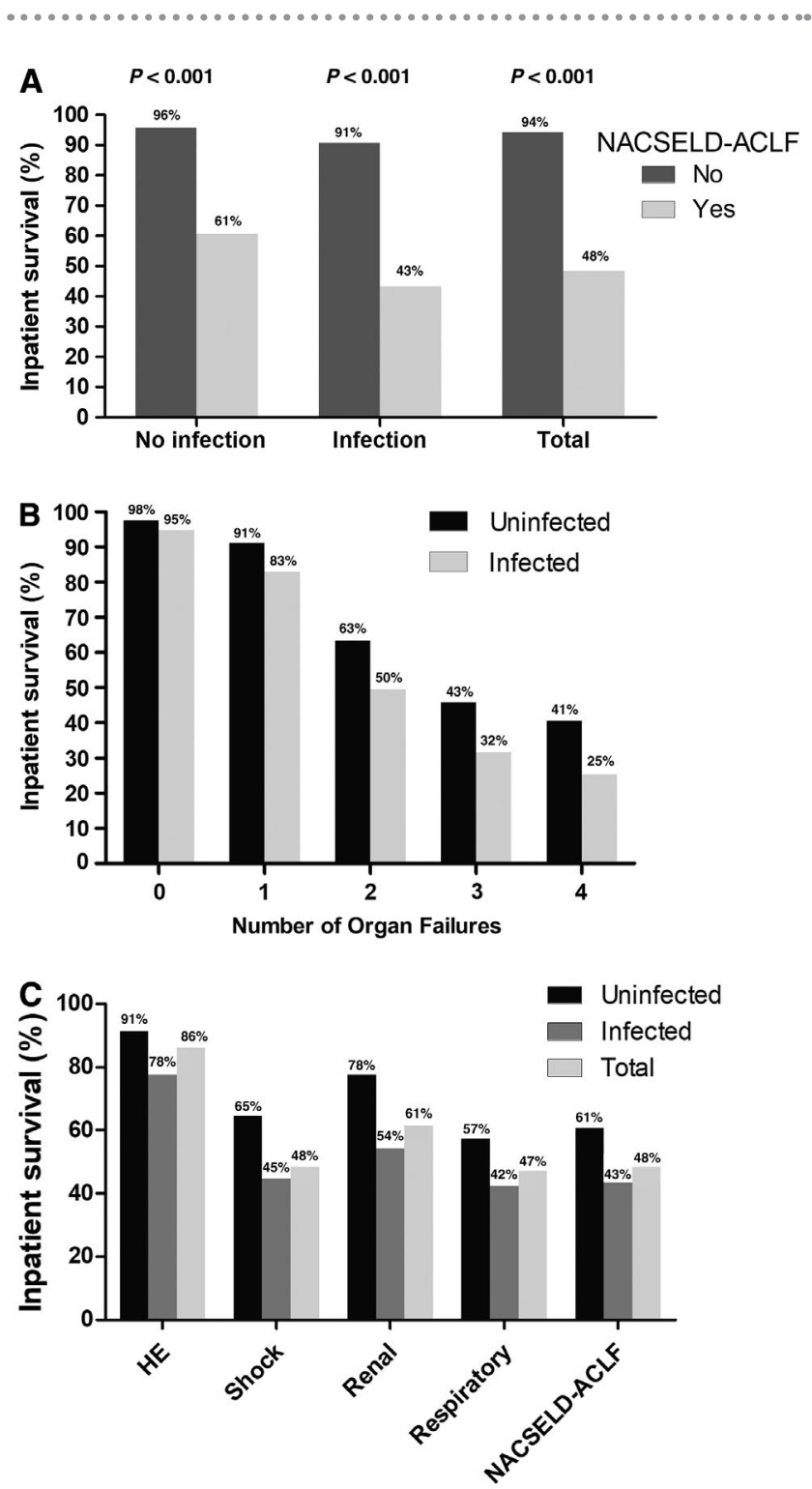

FIG. 2. Inpatient survival stratified by NACSELD-ACLF score, number of organ failures, and type of organ failure. (A) Inpatient survival by the presence of the NACSELD-ACLF score stratified by the presence of infection. (B) Inpatient survival by the number of organ failures stratified by the presence of infection. (C) Inpatient survival by the type of organ failure stratified by the presence of infection.

versus 59\%; $P<0.001)$ was higher in non-LT centers (Fig. 3B). On multivariate analysis, controlling for age, sex, the interaction of GI bleeding and presence of ascites, presence of infection, the Elixhauser comorbidity index, and NACSELD-ACLF score, hospitalization at an LT center was associated with increased survival (adjusted OR, 1.17; 95\% CI, 1.13-1.22; $P<0.001$; Table 4).
TABLE 2. Univariable Logistic Regression Survival Probability for Number of Organ Failures in the Entire Cohort

\begin{tabular}{lccccc} 
& \multicolumn{2}{c}{ Infection } & & \multicolumn{2}{c}{ No Infection } \\
\cline { 2 - 3 } \cline { 5 - 6 } Number of & Unadjusted OR Failures & $95 \% \mathrm{Cl}$ & Unadjusted OR & $95 \% \mathrm{Cl}$ \\
\hline No organ failure & Reference & \multicolumn{3}{c}{ Reference } \\
1 organ failure & 0.26 & $0.25-0.27$ & 0.26 & $0.25-0.27$ \\
2 organ failures & 0.05 & $0.05-0.05$ & 0.04 & $0.04-0.05$ \\
3 organ failures & 0.02 & $0.02-0.03$ & 0.02 & $0.02-0.03$ \\
4 organ failures & 0.02 & $0.01-0.02$ & 0.02 & $0.02-0.03$
\end{tabular}

TABLE 3. Multivariable Logistic Regression Survival Probability for NACSELD-ACLF Score in the Entire Cohort

\begin{tabular}{lccc} 
Variable & Adjusted OR & $95 \% \mathrm{Cl}$ & $P$ Value \\
\hline NACSELD-ACLF score & 0.08 & $0.07-0.08$ & $<0.001$ \\
Age & 0.98 & $0.98-0.99$ & $<0.001$ \\
Female & 1.20 & $1.16-1.23$ & $<0.001$ \\
Interaction of GI bleeding and & 0.78 & $0.73-0.84$ & $<0.001$ \\
$\quad$ ascites & & & \\
Infection & 0.42 & $0.41-0.43$ & $<0.001$ \\
Elixhauser comorbidity index & 0.990 & $0.979-0.996$ & 0.004
\end{tabular}

\section{Discussion}

The purpose of this study was to use a nationally representative database to externally validate the performance of NACSELD-ACLF score in predicting inpatient survival. We identified 1.5 million hospitalized patients with decompensated cirrhosis over 10 years and confirmed that organ failures were indeed a major driver of mortality in this population across the United States, particularly when the patient was infected. Furthermore, by selecting a population where the majority were hospitalized at non-LT centers, this analysis also validates the use of NACSELD-ACLF score among patients who may not have the option for LT.

The use of a database with national sampling in order to validate the NACSELD-ACLF score is crucial because the majority of patients in the United States are seen at nontransplant centers. Despite only one-sixth of the hospitalized population with cirrhosis being seen at academic LT centers, LT centers generate the vast majority of the literature advising inpatient cirrhosis management. Similar to this study, an analysis of the Veterans Administration database over a similar timeframe consisted of $<10 \%$ of patients hospitalized at LT centers, but this population was 

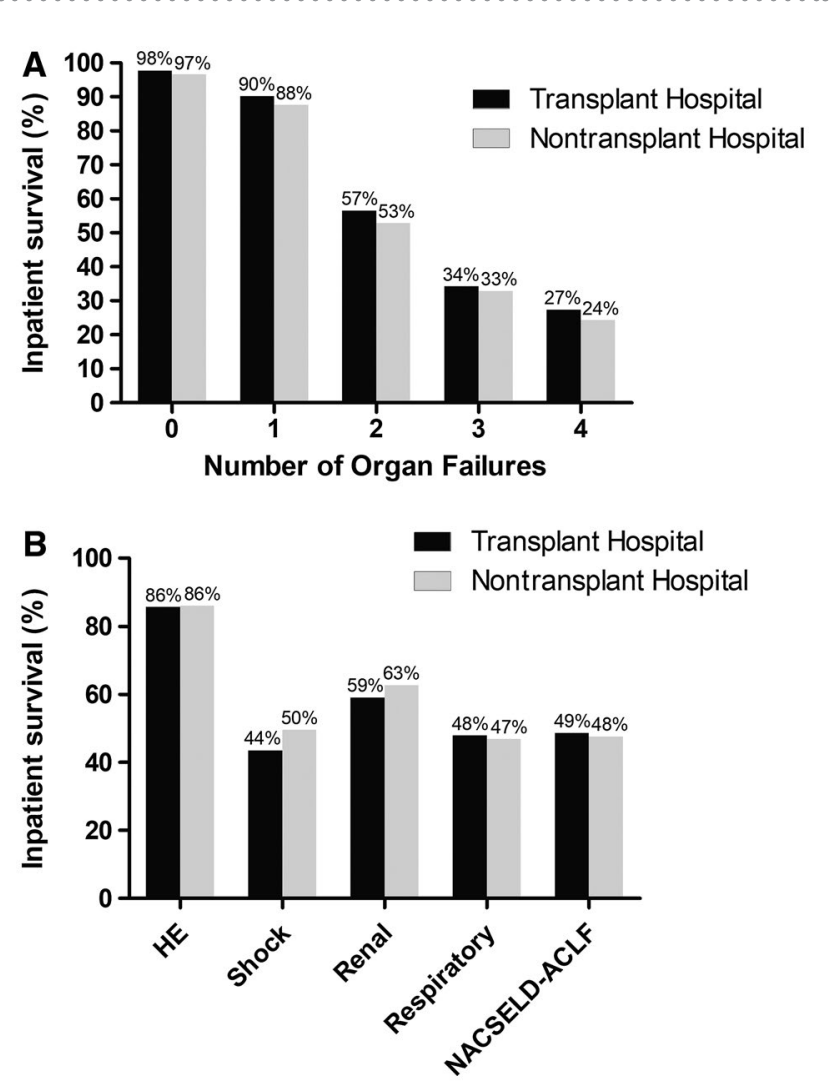

FIG. 3. Inpatient survival stratified by hospitalization at an LT center. (A) Inpatient survival by the number of organ failures depending on hospitalization in an LT center. (B) Inpatient survival by the type of organ failure depending on hospitalization in an LT center.

TABLE 4. Multivariable Logistic Regression Survival Probability for NACSELD-ACLF Score in the Entire Cohort With Inclusion of Hospitalization at a LT Center

\begin{tabular}{lccc} 
Variable & Adjusted OR & $95 \% \mathrm{Cl}$ & PValue \\
\hline Hospitalization at a LT center & 1.17 & $1.13-1.22$ & $<0.001$ \\
NACSELD-ACLF Score & 0.08 & $0.07-0.08$ & $<0.001$ \\
Age & 0.98 & $0.98-0.99$ & $<0.001$ \\
Female & 1.20 & $1.16-1.23$ & $<0.001$ \\
Interaction of Gl bleeding and & 0.79 & $0.73-0.84$ & $<0.001$ \\
$\quad$ ascites & & & \\
Infection & 0.42 & $0.41-0.43$ & $<0.001$ \\
Elixhauser comorbidity index & 0.990 & $0.981-0.998$ & 0.02
\end{tabular}

predominantly composed of male veterans, limiting its external validation. ${ }^{(15)}$ The NACSELD-ACLF score demonstrates its broadest appeal among patients and providers at non-LT hospitals. Patients seen in these hospitals do not have access to prompt salvage LT, and the presence of the NACSELD-ACLF score can quickly stratify these patients into a low $(<50 \%)$ and high $(>90 \%)$ chance of survival. These stark differences warrant a consideration of changing the goals of care when the NACSELD-ACLF score is present. Given the underuse of palliative care services in patients with liver disease who are denied LT, ${ }^{(16)}$ early identification of these patients would allow for a smoother transition to palliative care. For example, other diseases, most notably metastatic non-small cell lung cancer, have demonstrated not only improved quality of life and mood but also longer survival in patients who received early palliative care and avoided aggressive care. ${ }^{(17)}$ At the same time, patients who are potential transplant candidates, who meet the criteria of the NACSELDACLF score, and who are at a nontransplant hospital require urgent transfer to a transplant-capable hospital. The presence of the NACSELD-ACLF score can be used as an early indicator to initiate a timely transfer in a non-LT hospital or, similarly, warrant a rapid LT evaluation for a patient already in an LT hospital.

Although there were minor differences between the results of our analysis and the initial NACSELDACLF article $^{(6)}$ overall, the findings of both studies are quite similar. Survival for all organ failures was poor in both cohorts, but our cohort had notably reduced survival among patients with shock and especially with respiratory failure. Interestingly, we observed a significantly worse survival (10\% higher mortality) among patients with NACSELDACLF compared with the NACSELD study group cohort. ${ }^{(6)}$ Although there are no clear explanations for this discrepancy, it is likely driven by patients who are not LT candidates or a lack of inpatient liver services that are typical at major liver referral centers. Unsurprisingly, despite utilization of a different multivariate regression model, our study also found that NACSELD-ACLF was associated with a more potent effect on survival (OR, 0.08 versus 0.16 ). When comparing the impact of the NACSELDACLF score in the original and validation study, it showed itself in the original NACSELD-ACLF study to be simpler and more strongly linked with inpatient survival when compared with the MELD score (OR, 0.95). Notably, our analysis was also in agreement with O'Leary et al. ${ }^{(6)}$ in that infection was associated with significantly worse outcomes. This finding is in contrast to the CANONIC study, in which there were similar mortality rates among patients with and without infection. ${ }^{(4)}$ However, it 
is worth noting that infection was associated with ACLF, which, in the CANONIC study, was associated with poor outcomes.

The limitations of this study are inherent to the use of large databases in general, which is prone to administrative coding errors. Despite this limitation, the power of using 1.5 million hospitalizations is profound and is a tradeoff of administrative databases. The NIS specifically lacks laboratory data, which is crucial in determining liver disease severity, such as the calculation of Model for EndStage Liver Disease-sodium (MELD-Na) or ChildTurcotte-Pugh score. Furthermore, a key element within the NACSELD-ACLF multivariate model is white blood cell count, which was appreciated as an important marker of both inflammation and prognosis. ${ }^{(6)}$ Nonetheless, the model used in this study, although lacking laboratory covariates, included factors known to increase mortality in patients with cirrhosis. Because only 1 ICD-9-CM code was used to diagnose $\mathrm{HE}$, we were unable to differentiate between stages of $\mathrm{HE}$ severity. The inclusion of $\mathrm{HE}$ at all stages in our study likely explains why survival in our study is higher for patients with $\mathrm{HE}$ compared with the study by O'Leary et al. ${ }^{(6)}$ Unfortunately, because of poor coding algorithms, acute insults, such as alcoholic hepatitis and acute viral hepatitis, are challenging to diagnose and study individually when using a large database. ${ }^{(14)}$ Additionally, because this study is retrospective, it can merely confirm the findings demonstrated by the NACSELD-ACLF through association and is not, by itself, predictive of inpatient survival. Although the weaknesses of large databases are well documented, the external validity of this study is substantial given its broad representation of LT and non-LT hospitals in various locations in the country as compared with well-recognized liver referral hospitals included in the NACSELD study group. It is also worth noting that ACLF is a clinical syndrome that results in organ failures, in which laboratory values would be helpful but not fully necessary to diagnose.

Still, this analysis brings to light an increasingly important question: Does the NACSELDACLF score prior to LT impact post-LT survival? Unfortunately, although our database is limited in its ability to analyze the relationship between the timing of organ failures and LT, a recent analysis of the United Network for Organ Sharing database noted that despite the number of organ failures being associated with a worse posttransplant mortality, the absolute difference between those with no organ failures (90\% 1-year survival) and those with 5-6 organ failures (81\% 1-year survival) prior to LT, was only 9\%. ${ }^{(18)}$ Similar to the MELD-Na score, the NACSELD-ACLF score is excellent in predicting short-term mortality, but organ failures themselves do not necessarily indicate a poor posttransplant prognosis. In fact, patients meeting criteria for ACLF on the LT waiting list had higher mortality than those with acute liver failure, which was previously thought to confer the highest short-term mortality for patients with liver disease. ${ }^{(19)}$ In contrast to non-LT centers, LT centers should be incentivized to continue to consider patients with multiple organ failures for LT, especially when patients derive significant short-term and longterm survival benefit from LT. ${ }^{(20)}$ Prospective data from the NACSELD cohort demonstrate higher rates of pre-LT mortality in those with ACLF but no difference in post-LT survival among listed patients. ${ }^{(21)}$

However, retrospective studies have demonstrated mixed results. A study of patients with ACLF grade 3 from France had outcomes after LT similar to that of lower-grade ACLF, whereas other studies from France $^{(22)}$ and Germany ${ }^{(23)}$ show ACLF to be a potent risk factor for post-LT mortality. Currently, it is difficult to confidently predict post-LT mortality in patients who are entering LT with ACLF. Persistent renal failure after LT, which is a known adverse outcome after LT that is connected to mortality, may be a potential explanation for poor outcomes after LT in the setting of ACLF. ${ }^{(24)}$ Frailty, which is a major driver of wait-list mortality, ${ }^{(25)}$ is likely to be related to post-LT mortality as well. Future investigation is warranted because our study was limited by difficulties in interpreting timing of diagnoses and organ failures in relation to LT.

Given the rapid deterioration of patients with $\mathrm{ACLF}$, prediction of poor prognosis early in the time course is crucial. Our study now further supports the broad application of the NACSELD-ACLF score to identify patients hospitalized with ACLF, regardless of hospital type, and offers an easy bedside tool that patients, caregivers, and their providers can use to better recognize poor outcomes and enhance either early recognition of LT candidacy or, conversely, futile care in those without LT. In patients without access to LT who are very likely to die before discharge, the presence of the NACSELD-ACLF score predicted dismal survival; although on the contrary, a lack of 2 or more 
organ failures was associated with a $>94 \%$ inpatient survival. These findings represent a call to action for non-LT hospitals, who can use this simple bedside test to determine disposition in patients, depending on their transplant candidacy, to provide better quality care and outcomes.

\section{REFERENCES}

1) Sarin SK, Kedarisetty CK, Abbas $Z$, Amarapurkar D, Bihari $C$, Chan AC, et al.; for APASL ACLF Working Party. Acute-onchronic liver failure: consensus recommendations of the Asian Pacific Association for the Study of the Liver (APASL) 2014. Hepatol Int 2014;8:453-471.

2) Bajaj JS, Moreau R, Kamath PS, Vargas HE, Arroyo V, Reddy $\mathrm{KR}$, et al. Acute-on-chronic liver failure: getting ready for prime time? Hepatology 2018;68:1621-1632.

3) Jalan R, Yurdaydin C, Bajaj JS, Acharya SK, Arroyo V, Lin HC, et al.; for World Gastroenterology Organization Working Party. Toward an improved definition of acute-on-chronic liver failure. Gastroenterology 2014;147:4-10.

4) Moreau R, Jalan R, Gines P, Pavesi M, Angeli P, Cordoba J, et al.; for CANONIC Study Investigators of the EASL-CLIF Consortium. Acute-on-chronic liver failure is a distinct syndrome that develops in patients with acute decompensation of cirrhosis. Gastroenterology 2013;144:1426-1437.

5) Bajaj JS, O'Leary JG, Reddy KR, Wong F, Biggins SW, Patton H, et al.; for North American Consortium For The Study Of EndStage Liver Disease (NACSELD). Survival in infection-related acute-on-chronic liver failure is defined by extrahepatic organ failures. Hepatology 2014;60:250-256.

6) O'Leary JG, Reddy KR, Garcia-Tsao G, Biggins SW, Wong $\mathrm{F}$, Fallon MB, et al. NACSELD acute-on-chronic liver failure (NACSELD-ACLF) score predicts 30-day survival in hospitalized patients with cirrhosis. Hepatology 2018;67:2367-2374.

7) Mellinger JL, Richardson CR, Mathur AK, Volk ML. Variation among United States hospitals in inpatient mortality for cirrhosis. Clin Gastroenterol Hepatol 2015;13:577-584.

8) Nationwide Inpatient Sample (NIS), Healthcare Cost and Utilization Project, Agency for Healthcare Research and Quality. https://www.hcup-us.ahrq.gov/nisoverview.jsp. Accessed January 2020.

9) Goldberg D, Lewis J, Halpern S, Weiner M, Lo Re V3rd. Validation of three coding algorithms to identify patients with end-stage liver disease in an administrative database. Pharmacoepidemiol Drug Saf 2012;21:765-769.

10) Goto T, Yoshida K, Tsugawa Y, Filbin MR, Camargo CA Jr, Hasegawa K. Mortality trends in U.S. adults with septic shock, 2005-2011: a serial cross-sectional analysis of nationallyrepresentative data. BMC Infect Dis 2016;16:294.

11) Waikar SS, Wald R, Chertow GM, Curhan GC, Winkelmayer WC, Liangos O, et al. Validity of International Classification of
Diseases, Ninth Revision, Clinical Modification Codes for acute renal failure. J Am Soc Nephrol 2006;17:1688-1694.

12) Tapper EB, Halbert B, Mellinger J. Rates of and reasons for hospital readmissions in patients with cirrhosis: a multistate population-based cohort study. Clin Gastroenterol Hepatol 2016;14:1181-1188.

13) Elixhauser A, Steiner C, Harris DR, Coffey RM. Comorbidity measures for use with administrative data. Med Care 1998;36: 8-27.

14) Pang JX, Ross E, Borman MA, Zimmer S, Kaplan GG, Heitman SJ, et al. Validation of coding algorithms for the identification of patients hospitalized for alcoholic hepatitis using administrative data. BMC Gastroenterol 2015;15:116.

15) Hernaez R, Kramer JR, Liu Y, Tansel A, Natarajan Y, Hussain $\mathrm{KB}$, et al. Prevalence and short-term mortality of acute-on-chronic liver failure: a national cohort study from the USA. J Hepatol 2019;70:639-647.

16) Poonja Z, Brisebois A, van Zanten SV, Tandon P, Meeberg G, Karvellas CJ. Patients with cirrhosis and denied liver transplants rarely receive adequate palliative care or appropriate management. Clin Gastroenterol Hepatol 2014;12:692-698.

17) Temel JS, Greer JA, Muzikansky A, Gallagher ER, Admane S, Jackson VA, et al. Early palliative care for patients with metastatic non-small-cell lung cancer. N Eng1 J Med 2010;363:733-742.

18) Thuluvath PJ, Thuluvath AJ, Hanish S, Savva Y. Liver transplantation in patients with multiple organ failures: feasibility and outcomes. J Hepatol 2018;69:1047-1056.

19) Sundaram V, Shah P, Wong RJ, Karvellas CJ, Fortune BE, Mahmud N, et al. Patients with acute on chronic liver failure grade 3 have greater 14 -day waitlist mortality than status-1a patients. Hepatology 2019;70:334-345.

20) Merion RM, Schaubel DE, Dykstra DM, Freeman RB, Port FK, Wolfe RA. The survival benefit of liver transplantation. Am J Transplant 2005;5:307-313.

21) O'Leary JG, Bajaj JS, Tandon P, Biggins SW, Wong F, Kamath PS, et al. Outcomes after listing for liver transplant in patients with acute-on-chronic liver failure: the multicenter North American Consortium for the Study of End-Stage Liver Disease experience. Liver Transpl 2019;25:571-579.

22) Levesque E, Winter $A$, Noorah $Z$, Daurès JP, Landais $P$, Feray C, Azoulay D. Impact of acute-on-chronic liver failure on 90-day mortality following a first liver transplantation. Liver Int 2017;37:684-693.

23) Umgelter A, Lange K, Kornberg A, Büchler P, Friess H, Schmid RM. Orthotopic liver transplantation in critically ill cirrhotic patients with multi-organ failure: a single-center experience. Transplant Proc 2011;43:3762-3768.

24) Ojo AO, Held PJ, Port FK, Wolfe RA, Leichtman AB, Young $\mathrm{EW}$, et al. Chronic renal failure after transplantation of a nonrenal organ. N Engl J Med 2003;349:931-940.

25) Lai JC, Rahimi RS, Verna EC, Kappus MR, Dunn MA, McAdams-DeMarco M, et al. Frailty associated with waitlist mortality independent of ascites and hepatic encephalopathy in a multicenter study. Gastroenterology 2019;156:1675-1682. 